\title{
Covid-19 forecasting in Morocco using FBprophet Facebook's Framework in Python
}

\author{
Tarik CHAFIQ ${ }^{1}$, Mohammed OUADOUD ${ }^{2}$, Khadija ELBOUKHARI ${ }^{3}$ \\ ${ }^{1}$ Faculty of sciences Ben M'sik-University Hassan II, Casablanca, Morocco, Tarik.chafiq1@ gmail.com \\ ${ }^{2}$ Abdelmalek Essaadi University (UAE), Morocco, mohammed.ouadoud@ gmail.com \\ ${ }^{3}$ Sidi Mohamed Ben Abdellah University, University Hospital (CHU) of Fez, Morocco, \\ elboukharikhadija89@gmail.com
}

\begin{abstract}
Coronavirus Disease COVID-19 is a virus identified as the cause of an outbreak of severe acute respiratory syndrome detected first in Wuhan, China, On 31 December 2019. It has already taken a pandemic form, affecting over 200 countries in a few months. On March 3rd, 2020, the Moroccan Minister of Health announced the first case of coronavirus on March 2nd in Morocco, which was a Moroccan citizen who was returning from a trip to Italy. Then the pandemic spread has grown exponentially with more than 100,000 confirmed cases as of September 23th, 2020. After the partial lifting of containment in Morocco, the number of new cases reported daily is rising day by day. This study gives an indication of forecasting the spread of COVID-19 in the coming 4 months by Facebook Framework (FBprophet) using in python. This might be helpful for decision-makers and health authorities to evaluate risks and take proper precautions and strategies to minimize the spread of infectious diseases.
\end{abstract}

Key words: COVID-19, Framework, Prediction, Morocco, FBprophet

\section{INTRODUCTION}

Coronavirus is one of the major microorganisms that targeted primarily the human respiratory system with a high public health treat. It was responsible for two outbreaks including the severe acute respiratory syndrome (SARS)-CoV and the Middle East respiratory syndrome (MERS)-Cov [1].

On December 31st, China has reported to the World Health Organization (WHO) a severe acute respiratory syndrome of unknown etiology in Wuhan [2,3], which later turned to be Coronavirus SARS-CoV-2, the most contagious and quickly spreading entity of the Corona Virus family[1] which is locking up billions of people[4]. Coronavirus disease (COVID-19) as scientifically named by the World Health Organization in Feb [5] The first cases of Coronavirus was identified in Wuhan in December 2019 [6] in a cluster of patients sharing a history of visiting the seafood and wet animals market of Wuhan [7], which suggest the likely zoonotic origin of this disease [1] . after incubation of 5,2 days approximatively, symptoms appear and are dominated by fever, cough, and fatigue, while other symptoms include respiratory involvement such are sputum production, head-ache, hemoptysis dyspnea, diarrhea, and hematological involvement(lymphopenia)[8-10]. Most people affected with the COVID-19 are elderly [11], patients with underlying medical problems like cardiovascular disease, diabetes, chronic respiratory disease. Those people are more likely to develop serious illnesses.

With 37,756,558 world confirmed cases and 1,081,528 confirmed deaths $(\sim 3 \%)$ as on 12th October 2020 [12], COVID-19 has already surpassed the previous records for two coronaviruses SARS-CoV and MERS-CoV, which represents the biggest threat to the health and world economy after the second world war [13].

Morocco is not far behind the pandemic. On March 3rd, 2020, the Minister of Health announced the first case of coronavirus detected on March 2nd in Morocco [14]; a Moroccan citizen who was returning from a trip to Italy. Then, On March 16th, 2020, the Moroccan Government was implemented measures to slow and limit the spread of viral transmission of COVID-19 by adopting the following measures:

School closures

Maintaining social distancing

Bans on events and gatherings

Suspension of cultural, educational, sporting activities Suspending all public traffics within the city on 21th March 2020 except for freight transport

Despite the best efforts, certainly important, on September 23th, 2020 the Moroccan ministry of public health [15] and Maghreb Arab Press of Morocco (MAP) [16] announced a total of 107,743 confirmed cases of infection with COVID-19 and a total of 1918 deaths ( 2\%).

A few days after the announcement, on June 19th, 2020, of the lifting of containment, several epidemiological foci of the coronavirus appeared in certain units specializing in the packaging of fruits in the province of Kenitra, as well as in 
industrial circles in Tangier as the biggest number of the confirmed case till now, more than 500 cases have been recorded in one fell swoop, compared to an average of about 100 in containment. This is the highest daily toll since the announcement of the first contamination. For the moment, the situation is not worrying because they are not scattered cases. But the second wave is not immune, because the number of tests increased, the factories are opened, economic activity is resuming, so it would not be surprising. There is a risk of a relapse and a second wave of the pandemic's spread given the current unprecedented health crisis. The second wave could, in theory, be more important than the first, since many people are still sensitive. The prospect of a second wave of Covid-19 in Morocco is not excluded.

Daily information about affected people can provide valuable information[17] when it is made available for the data scientist community to analyze and provides forecasting for decision-makers, which will allow them to deal with the situation properly. Indeed, the model used in this prediction study presents a margin of error due to the influence of the presence of effective treatment and/or vaccine can influence this prediction.

\section{MATERIALS AND METHODS}

\subsection{Experimental Data}

The dataset has daily level information on the number of affected cases by COVID-19, the period starts on the 02nd of March 2020 and ends on the 29th of August 2020. It must be noted that this period was characterized by daily data regarding confirmed cases in Morocco on any given day which is a cumulative number. The data is freely available and has been extracted through the data repository maintained by the Johns Hopkins University[18].

\subsection{FBprophet framework}

FBProphet is an open-source forecasting framework developed by Facebook's team data scientist. It is used for forecasting time series data based on an additive model which can make the task of forecasting more accessible and easier to carry out. It works best with time series that have strong seasonal effects and several seasons of historical data[19]. FBProphet uses ARIMA, exponential models, and other similar regressive models.

The time series which is wanted to be predicted by using FBprophet [19] in Python is the number of accumulated confirmed cases (C) of COVID-19 in Morocco. The idea is that historical time series records can be used to forecast the values of the four coming months. Moreover, the FBprophet is based on decomposable time-series combined in this equation

$$
y(t)=g(t)+s(t)+h(t)+\varepsilon t
$$

and which consists of three main components:

- $g(t)$ : represents the trend function; piecewise linear or logical growth to fit non-periodic changes in the value of the time series
- $s(t)$ : represents the periodic changes as a week and/or year seasonality

- $h(t)$ : represents the effects of holidays that occur on irregular schedules over a day or more.

- $\varepsilon t$ : represents any unusual change which is not accommodated by the model.

\subsection{Solution method}

Open source tools in optimization and performance have grown in popularity over the last years[20,21] and can be observed in works like the optimization process in supply chain management [20,22] and machine learning applications. The approach adopted for forecasting the spread of COVID-19 in Morocco using open-source is represented in this study by BFprophet. The basics steps of the prediction procedure method of our model are shown in figure 1:

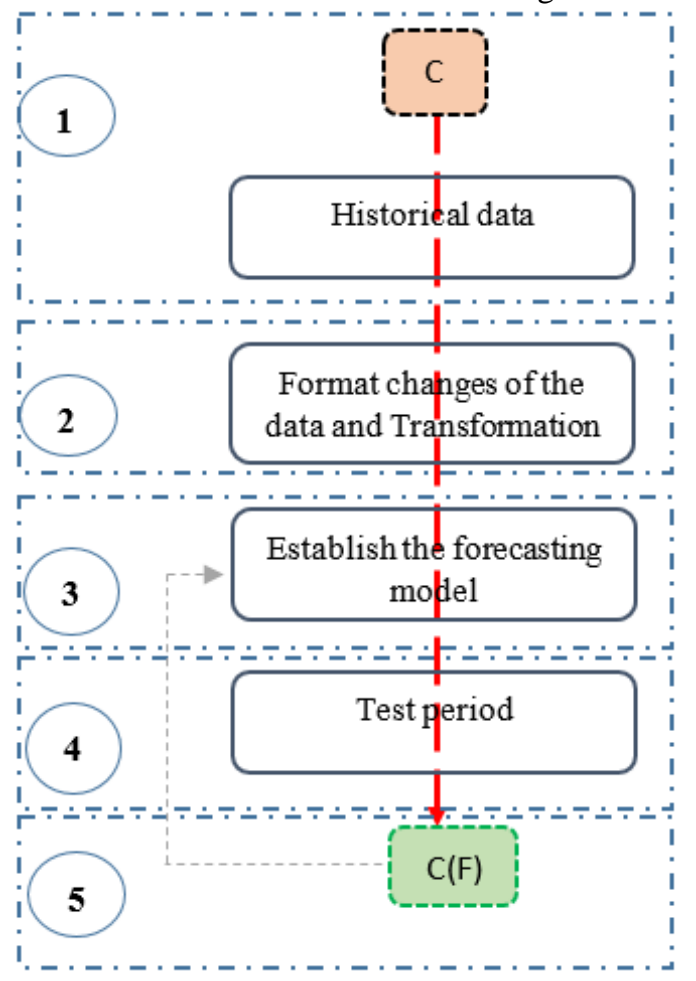

Figure 1: prediction procedure method

1- Daily collect historical time series records announced by the Ministry of Public Health of Morocco [15] and Maghreb Arab Press of Morocco (MAP)[16].

2- Since the dataset is made of daily records, they need to be converted to date instead of a string format. Then the transformation of the Confirmed cases should be applied by logarithmic function to be linear.

3- Based on the historical dataset, the model will be fitted by the framework of FBprophet.

4- According to the forecasting model fitted a suitable algorithm generated to predict the defined period. 
5- The results of Confirmed cases forecasting $\mathrm{C}(\mathrm{F})$ depend on the quantity and quality of the dataset injected and fitted in the model to forecast. In particular, we assume that the average frequency and magnitude of trend changes in the future will be the same as that which we observe in the historical time series.

\section{RESULTS}

The predicted fit model based on the transformed number of confirmed cases by the logarithm(C), has generated a quite well plot prediction (figure 2) for the coming days.

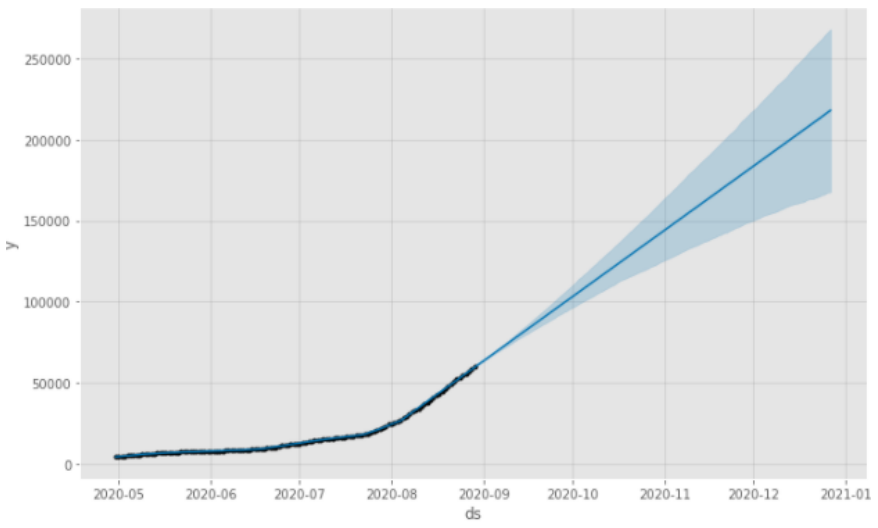

Figure 2: Forecasting Plot of the coming days by Log

Moreover, the framework of FBprophet will create a new dataset (APPENDIX 1) assigned to the forecast variable that contains the forecasted values for future dates under the column yhat which represent forecasting based on linear regression, as well as an interval in both ways', a sigmoid (yhat_lower) curve as the second and desirable scenario if the citizens respect the instructions of the Moroccan authorities, otherwise, an exponential (yhat_upper) curve will be followed as the third no desirable scenario. This means that many confirmed cases can vary from lowest to highest.

The forecast plot (figure 3) draws the original data (red dots and line), the forecasting model (blue dots and line), and the two scenarios of the forecast (shaded blue area).

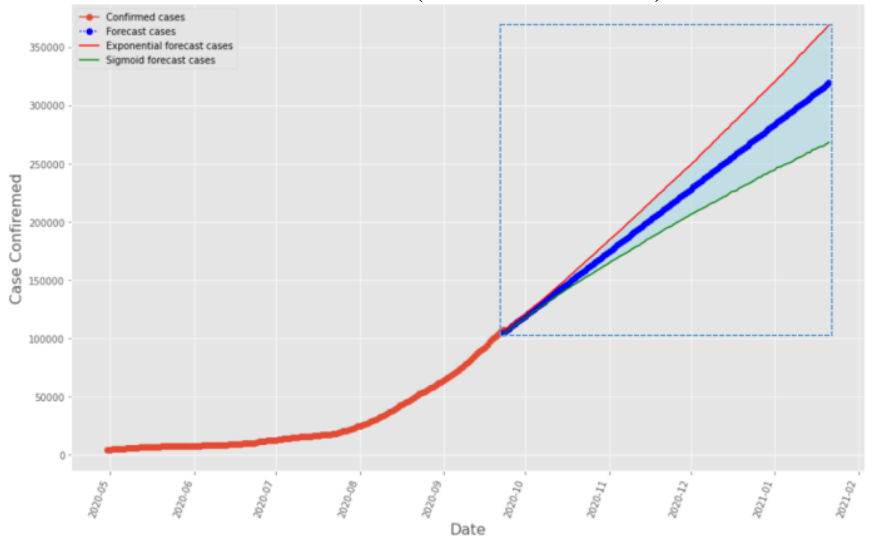

Figure 3: Forecast plot including Confirmed cases, forecast confirmed cases and uncertainty intervals
The forecasting model and component (figure 4) visualizations show the underlying trend in the predicted trend as a linear trend, while also accurately modeling weekly seasonality (e.g. upper confirmed cases on Saturday and Sunday). This trend analysis can be used to effectively shows where to led resources up to weeks and months in the future on the pandemic.
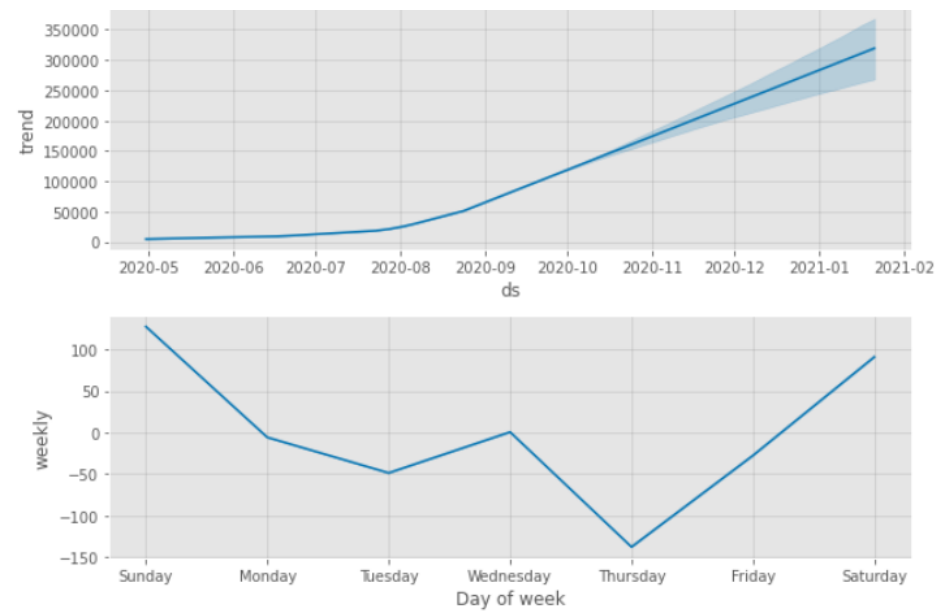

Figure 4: The trend and the weekly seasonality plot

\section{MODEL VALIDATION}

In order to find out how the model performs some form of validation was needed. Fortunately, the Prophet library makes it possible to divide our historical data into training data and testing data for cross validation.

The resulting dataset can now be used to compute error measures of yhat aforementioned, which was plotted as a chart with some legend to help understanding in a more visual way and gives as a results of validation (Table 1) using the statistics computed are mean squared error (MSE), root mean squared error (RMSE), mean absolute error (MAE), mean absolute percent error (MAPE), and coverage of the yhat_lower and yhat_upper estimates.

Table 1: Listing the performance data frame gives us the following

\begin{tabular}{|c|c|c|c|c|c|c|}
\hline Horizon & Mse & Rmse & Mae & Mape & Mdape & Coverage \\
\hline 9 days & $6.405898 \mathrm{e}+05$ & 800.368528 & 776.513857 & 0.061254 & 0.056904 & 0.0 \\
\hline 10 days & $8.424479 \mathrm{e}+05$ & 917.849624 & 873.389707 & 0.067018 & 0.060468 & 0.0 \\
\hline 11 days & $1.031520 \mathrm{e}+06$ & 1015.637489 & 963.423658 & 0.072229 & 0.061642 & 0.0 \\
\hline 12 days & $1.239342 \mathrm{e}+06$ & 1113.257587 & 1059.578427 & 0.077767 & 0.069815 & 0.0 \\
\hline 13 days & $1.419011 \mathrm{e}+06$ & 1191.222471 & 1143.122350 & 0.082361 & 0.090917 & 0.0 \\
\hline 14 days & $1.623446 \mathrm{e}+06$ & 1274.145392 & 1230.728643 & 0.087015 & 0.098249 & 0.0 \\
\hline 15 days & $1.873432 \mathrm{e}+06$ & 1368.733902 & 1340.182119 & 0.093153 & 0.101200 & 0.0 \\
\hline 16 days & $2.105210 \mathrm{e}+06$ & 1450.934258 & 1435.655411 & 0.098183 & 0.103522 & 0.0 \\
\hline
\end{tabular}




\section{CONCLUSION}

This study provides a trend mechanism as seen by FBprophet of pandemics of coronavirus (COVID-19) spread from 23th September 2020 to 21th January 2020, which can provide valuable insight for the national competent authorities; In future research, we plan to pair this research with Deep Learning models such as the Deep Sequential Prediction Model (DSPM) and compare it with the FBprophet result's to refine the prediction models to further improve the prediction of the spread of this disease. 
Tarik CHAFIQ et al., International Journal of Advanced Trends in Computer Science and Engineering, 9(5),September-October 2020, 8654 - 8660

\section{APPENDIX}

APPENDIX 1: Evolution of the coronavirus in the coming days in morocco

\begin{tabular}{|c|c|c|c|}
\hline OBSERVATION & YHAT & YHAT_LOWER & YHAT_UPPER \\
\hline $23 / 09 / 2020$ & 104841.782 & 103409.859 & 106359.748 \\
\hline $24 / 09 / 2020$ & 106490.238 & 105160.897 & 107888.699 \\
\hline $25 / 09 / 2020$ & 108387.162 & 106883.298 & 109704.035 \\
\hline $26 / 09 / 2020$ & 110292.612 & 108796.223 & 111738.901 \\
\hline $27 / 09 / 2020$ & 112115.975 & 110650.907 & 113493.245 \\
\hline $28 / 09 / 2020$ & 113769.399 & 112300.570 & 115267.931 \\
\hline $29 / 09 / 2020$ & 115513.375 & 114090.874 & 117134.935 \\
\hline $30 / 09 / 2020$ & 117349.409 & 115808.327 & 118882.885 \\
\hline $01 / 10 / 2020$ & 118997.865 & 117374.465 & 120582.509 \\
\hline $02 / 10 / 2020$ & 120894.789 & 119027.173 & 122634.255 \\
\hline 03/10/2020 & 122800.239 & 121034.147 & 124592.609 \\
\hline $04 / 10 / 2020$ & 124623.602 & 122700.594 & 126522.325 \\
\hline 05/10/2020 & 126277.026 & 124263.612 & 128426.091 \\
\hline $06 / 10 / 2020$ & 128021.002 & 125762.357 & 130277.609 \\
\hline $07 / 10 / 2020$ & 129857.036 & 127632.299 & 132320.793 \\
\hline $08 / 10 / 2020$ & 131505.492 & 128892.723 & 134185.742 \\
\hline 09/10/2020 & 133402.416 & 130700.969 & 136289.392 \\
\hline $10 / 10 / 2020$ & 135307.866 & 132366.024 & 138233.193 \\
\hline $11 / 10 / 2020$ & 137131.229 & 133945.821 & 140386.130 \\
\hline $12 / 10 / 2020$ & 138784.653 & 135419.031 & 142397.426 \\
\hline $13 / 10 / 2020$ & 140528.629 & 136921.732 & 144312.403 \\
\hline $14 / 10 / 2020$ & 142364.663 & 138389.758 & 146401.699 \\
\hline $15 / 10 / 2020$ & 144013.119 & 139766.206 & 148275.560 \\
\hline $16 / 10 / 2020$ & 145910.043 & 141575.488 & 150274.648 \\
\hline $17 / 10 / 2020$ & 147815.493 & 142962.352 & 152687.827 \\
\hline $18 / 10 / 2020$ & 149638.856 & 144580.887 & 154723.427 \\
\hline $19 / 10 / 2020$ & 151292.280 & 145908.213 & 156668.895 \\
\hline $20 / 10 / 2020$ & 153036.256 & 147466.855 & 159045.002 \\
\hline $21 / 10 / 2020$ & 154872.290 & 148799.002 & 161086.628 \\
\hline $22 / 10 / 2020$ & 156520.746 & 150206.366 & 162877.521 \\
\hline $23 / 10 / 2020$ & 158417.670 & 151990.569 & 165329.782 \\
\hline $24 / 10 / 2020$ & 160323.120 & 153163.108 & 167224.483 \\
\hline $25 / 10 / 2020$ & 162146.483 & 154488.205 & 169381.452 \\
\hline $26 / 10 / 2020$ & 163799.907 & 155971.124 & 171179.968 \\
\hline $27 / 10 / 2020$ & 165543.883 & 157467.571 & 173310.752 \\
\hline $28 / 10 / 2020$ & 167379.917 & 158865.892 & 175840.639 \\
\hline $29 / 10 / 2020$ & 169028.373 & 160199.616 & 177808.744 \\
\hline $30 / 10 / 2020$ & 170925.297 & 161936.506 & 179856.523 \\
\hline $31 / 10 / 2020$ & 172830.747 & 163022.125 & 181939.429 \\
\hline $01 / 11 / 2020$ & 174654.110 & 164597.725 & 184474.878 \\
\hline
\end{tabular}

\begin{tabular}{|c|c|c|c|}
\hline $02 / 11 / 2020$ & 176307.534 & 166421.475 & 186231.893 \\
\hline $03 / 11 / 2020$ & 178051.510 & 167541.162 & 188283.175 \\
\hline $04 / 11 / 2020$ & 179887.544 & 168853.149 & 190254.363 \\
\hline $05 / 11 / 2020$ & 181536.000 & 170532.590 & 192373.442 \\
\hline $06 / 11 / 2020$ & 183432.924 & 171512.309 & 194421.214 \\
\hline $07 / 11 / 2020$ & 185338.374 & 172859.098 & 196859.689 \\
\hline $08 / 11 / 2020$ & 187161.737 & 174086.704 & 199179.796 \\
\hline $09 / 11 / 2020$ & 188815.161 & 175885.656 & 200983.551 \\
\hline $10 / 11 / 2020$ & 190559.137 & 177405.943 & 203201.808 \\
\hline $11 / 11 / 2020$ & 192395.171 & 178797.944 & 205581.850 \\
\hline $12 / 11 / 2020$ & 194043.627 & 179577.650 & 207464.020 \\
\hline $13 / 11 / 2020$ & 195940.551 & 181483.788 & 209503.974 \\
\hline $14 / 11 / 2020$ & 197846.001 & 182777.821 & 212126.505 \\
\hline $15 / 11 / 2020$ & 199669.364 & 184808.046 & 214330.795 \\
\hline $16 / 11 / 2020$ & 201322.788 & 185821.159 & 216141.510 \\
\hline $17 / 11 / 2020$ & 203066.764 & 187268.111 & 218738.453 \\
\hline $18 / 11 / 2020$ & 204902.798 & 188496.861 & 220620.904 \\
\hline 19/11/2020 & 206551.254 & 189778.696 & 223185.077 \\
\hline $20 / 11 / 2020$ & 208448.178 & 191139.084 & 225089.103 \\
\hline $21 / 11 / 2020$ & 210353.628 & 192805.465 & 226888.411 \\
\hline $22 / 11 / 2020$ & 212176.991 & 194379.732 & 229824.296 \\
\hline $23 / 11 / 2020$ & 213830.415 & 195286.240 & 231834.075 \\
\hline $24 / 11 / 2020$ & 215574.391 & 196252.199 & 233950.234 \\
\hline $25 / 11 / 2020$ & 217410.425 & 197968.887 & 235856.233 \\
\hline $26 / 11 / 2020$ & 219058.881 & 199410.855 & 238220.539 \\
\hline $27 / 11 / 2020$ & 220955.805 & 200650.466 & 240048.316 \\
\hline 28/11/2020 & 222861.255 & 202018.203 & 242586.457 \\
\hline $29 / 11 / 2020$ & 224684.618 & 203404.451 & 244733.000 \\
\hline $30 / 11 / 2020$ & 226338.042 & 204620.075 & 246507.643 \\
\hline $01 / 12 / 2020$ & 228082.018 & 206153.233 & 248598.661 \\
\hline $02 / 12 / 2020$ & 229918.052 & 207295.588 & 251161.416 \\
\hline $03 / 12 / 2020$ & 231566.508 & 208603.097 & 252648.516 \\
\hline $04 / 12 / 2020$ & 233463.432 & 209931.148 & 255713.808 \\
\hline $05 / 12 / 2020$ & 235368.882 & 210863.513 & 258193.683 \\
\hline $06 / 12 / 2020$ & 237192.245 & 212081.015 & 260498.129 \\
\hline $07 / 12 / 2020$ & 238845.669 & 213265.507 & 262677.434 \\
\hline $08 / 12 / 2020$ & 240589.645 & 215077.418 & 264402.432 \\
\hline $09 / 12 / 2020$ & 242425.679 & 216200.580 & 267132.823 \\
\hline $10 / 12 / 2020$ & 244074.135 & 216835.261 & 269690.895 \\
\hline $11 / 12 / 2020$ & 245971.059 & 218366.675 & 271631.370 \\
\hline $12 / 12 / 2020$ & 247876.509 & 219747.382 & 274380.221 \\
\hline $13 / 12 / 2020$ & 249699.872 & 221128.410 & 276441.660 \\
\hline $14 / 12 / 2020$ & 251353.296 & 221822.866 & 278955.483 \\
\hline
\end{tabular}




\begin{tabular}{|c|c|c|c|}
\hline $15 / 12 / 2020$ & 253097.272 & 223650.741 & 280333.650 \\
\hline $16 / 12 / 2020$ & 254933.306 & 225630.286 & 283847.729 \\
\hline $17 / 12 / 2020$ & 256581.763 & 226032.513 & 285617.843 \\
\hline $18 / 12 / 2020$ & 258478.686 & 226722.806 & 287474.265 \\
\hline $19 / 12 / 2020$ & 260384.136 & 228920.857 & 290100.326 \\
\hline 20/12/2020 & 262207.499 & 229987.146 & 292361.787 \\
\hline 21/12/2020 & 263860.923 & 231254.641 & 294437.568 \\
\hline $22 / 12 / 2020$ & 265604.899 & 232131.775 & 297244.287 \\
\hline $23 / 12 / 2020$ & 267440.933 & 233342.461 & 299496.881 \\
\hline $24 / 12 / 2020$ & 269089.390 & 234605.256 & 301372.064 \\
\hline $25 / 12 / 2020$ & 270986.313 & 236235.531 & 303547.666 \\
\hline 26/12/2020 & 272891.763 & 237949.654 & 306943.131 \\
\hline 27/12/2020 & 274715.126 & 238370.694 & 308568.291 \\
\hline $28 / 12 / 2020$ & 276368.550 & 240497.649 & 311039.729 \\
\hline 29/12/2020 & 278112.526 & 240955.766 & 313058.461 \\
\hline $30 / 12 / 2020$ & 279948.560 & 242559.365 & 315630.300 \\
\hline $31 / 12 / 2020$ & 281597.017 & 243565.968 & 317870.422 \\
\hline $01 / 01 / 2021$ & 283493.940 & 244207.422 & 319868.880 \\
\hline $02 / 01 / 2021$ & 285399.390 & 246783.026 & 322512.274 \\
\hline $03 / 01 / 2021$ & 287222.753 & 246917.452 & 324959.543 \\
\hline $04 / 01 / 2021$ & 288876.177 & 248153.345 & 326734.513 \\
\hline $05 / 01 / 2021$ & 290620.153 & 249383.625 & 329642.885 \\
\hline 06/01/2021 & 292456.187 & 250123.616 & 331928.797 \\
\hline $07 / 01 / 2021$ & 294104.644 & 250842.364 & 334234.272 \\
\hline $08 / 01 / 2021$ & 296001.568 & 252326.180 & 336366.330 \\
\hline 09/01/2021 & 297907.017 & 254208.322 & 339145.222 \\
\hline $10 / 01 / 2021$ & 299730.380 & 255086.510 & 342577.714 \\
\hline $11 / 01 / 2021$ & 301383.804 & 255966.647 & 344570.466 \\
\hline $12 / 01 / 2021$ & 303127.780 & 257236.277 & 346052.128 \\
\hline $13 / 01 / 2021$ & 304963.814 & 259210.880 & 349321.921 \\
\hline $14 / 01 / 2021$ & 306612.271 & 259362.057 & 351743.900 \\
\hline $15 / 01 / 2021$ & 308509.195 & 261750.592 & 354237.187 \\
\hline $16 / 01 / 2021$ & 310414.644 & 262235.786 & 356354.743 \\
\hline $17 / 01 / 2021$ & 312238.007 & 263557.703 & 359269.981 \\
\hline $18 / 01 / 2021$ & 313891.431 & 264523.543 & 361172.635 \\
\hline $19 / 01 / 2021$ & 315635.407 & 264793.778 & 363535.168 \\
\hline $20 / 01 / 2021$ & 317471.441 & 266610.038 & 366196.311 \\
\hline $21 / 01 / 2021$ & 319119.898 & 267953.638 & 368726.952 \\
\hline
\end{tabular}

\section{ACKNOWLEDGE}

The authors extend much gratitude to the Editorial office and peer reviewers

\section{CONFLICT OF INTEREST STATEMENTS}

The authors declare no competing interests.

\section{FUNDING}

This research received no specific grant from any funding agency in the public, commercial, or not-for-profit sectors.

\section{AVAILABILITY OF DATA AND MATERIAL}

All data generated or analysed during this study are freely available through the data repository maintained by the Johns Hopkins University

\section{AUTHORS' CONTRIBUTIONS}

Authors contributed equally to this manuscript

\section{REFERENCES}

1. Rothan, H.A. and S.N. Byrareddy, The epidemiology and pathogenesis of coronavirus disease (COVID-19) outbreak. Journal of Autoimmunity, 2020: p. 102433.

2. Jiang, S., et al., A novel coronavirus (2019-nCoV) causing pneumonia-associated respiratory syndrome. Cellular \& molecular immunology, 2020: p. 1-1.

3. Dheeraj, K., Analysing COVID-19 news impact on social media aggregation. International Journal of Advanced Trends in Computer Science and Engineering, 2020. 9(3).

4. Ibrahim, S., Kamaruddin, S.A., Mangshor, N.N., Fadzil, A.F.A., Performance evaluation of multi-layer perceptron (MLP) and radial basis function (RBF): COVID-19 spread and death contributing factors. International Journal of Advanced Trends in Computer Science and Engineering, 2020. 9(1.4): p. 625-631.

5. WHO. Naming the coronavirus disease (COVID-19) and the virus that causes it. 2019 [cited 2020 30-08-2020]; Available from: https://www.who.int/emergencies/diseases/novel-cor onavirus-2019/technical-guidance/naming-the-coron avirus-disease-(covid-2019)-and-the-virus-that-caus es-it.

6. Du Toit, A., Outbreak of a novel coronavirus. Nature Reviews Microbiology, 2020. 18(3): p. 123-123.

7. Bogoch, I.I., et al., Pneumonia of Unknown Etiology in Wuhan, China: Potential for International Spread Via. 2020.

8. Huang, C., et al., Clinical features of patients infected with 2019 novel coronavirus in Wuhan, China. The Lancet, 2020. 395(10223): p. 497-506.

9. Wang, W., J. Tang, and F. Wei, Updated understanding of the outbreak of 2019 novel coronavirus (2019-nCoV) in Wuhan, China. Journal of medical virology, 2020. 92(4): p. 441-447. 
10. Carlos, W.G., et al., Novel wuhan (2019-nCoV) coronavirus. American journal of respiratory and critical care medicine, 2020. 201(4): p. P7-P8.

11. Chen, N., et al., Epidemiological and clinical characteristics of 99 cases of 2019 novel coronavirus pneumonia in Wuhan, China: a descriptive study. The Lancet, 2020. 395(10223): p. 507-513.

12. (WHO), W.H.O. Coronavirus disease (COVID-2019) situation reports. 2020 [cited 2020 12-10-2020]; Available from: https://www.who.int/emergencies/diseases/novel-cor onavirus-2019/situation-reports/.

13. Paul, A., S. Chatterjee, and N. Bairagi, Prediction on Covid-19 epidemic for different countries: Focusing on South Asia under various precautionary measures. medRxiv, 2020.

14. MAP. Coronavirus: Des chambres affectées aux hôpitaux du Royaume pour recevoir les cas suspects. 2020 [cited 2020 01-04-2020]; Available from:

https://www.mapnews.ma/fr/actualites/social/corona virus-des-chambres-affect\%C3\%A9es-aux-h\%C3\% B4pitaux-du-royaume-pour-recevoir-les-cas.

15. Morocco, M.o.P.H.o. Le Portail Officiel du Coronavirus au Maroc. 2020; Available from: http://www.covidmaroc.ma/pages/Accueil.aspx.

16. MAP. La MAP EN LUTTE CONTRE LE COVID-19. 2020 [cited 2020 23-09-2020]; Available from: http://mapanticorona.map.ma/fr.

17. Chafiq, T., et al. Towards SDI Services for Geological Maps Data. In: International Conference on Advanced Intelligent Systems for Sustainable Development, 2018. Springer, pp 34-44. doi:https://doi.org/10.1007/978-3-030-11881-5_4

18. Dong, E., H. Du, and L. Gardner, An interactive web-based dashboard to track COVID-19 in real time. The Lancet Infectious Diseases, 2020.

19. Taylor, S.J. and B. Letham, Forecasting at scale. The American Statistician, 2018. 72(1): p. 37-45.

20. Romero-Gelvez, J.I., E.A. Delgado-Sierra, and J. Aurelio, Demand Forecasting and Material Requirement Planning Optimization using Open Source Tools. 2019.

21. Chafiq, T., et al. (2018) Application of data integrity algorithm for geotechnical data quality management doi:https://doi.org/10.3991/ijim.v12i8.9569

22. Moreno, R.H. and O. Garcia. Model of neural networks for fertilizer recommendation and amendments in pasture crops. in 2018 ICAI Workshops (ICAIW). 2018. IEEE. 